\title{
Magnetic and orbital blocking in Ni nanocontacts
}

\author{
David Jacob, J. Fernández-Rossier, and J. J. Palacios \\ Departamento de Física Aplicada, Universidad de Alicante, 03690 San Vicente del Raspeig, Spain
}

(Received 11 April 2005; published 9 June 2005)

\begin{abstract}
We address the fundamental question of whether magnetoresistance (MR) of atomic-sized contacts of nickel is very large because of the formation of a domain wall (DW) at the neck. Using ab initio transport calculations we find that, as in the case of nonmagnetic electrodes, transport in Ni nanocontacts depends very much on the orbital nature of the electrons. Our results are in agreement with several experiments in the average value of the conductance. On the other hand, contrary to existing claims, DW scattering does not account for large MR in Ni nanocontacts.
\end{abstract}

DOI: 10.1103/PhysRevB.71.220403

PACS number(s): 75.47.Jn, 75.50.Cc, 73.63.Rt

The strong sensitivity of the current flow between two ferromagnetic metals (FM's) separated by a nonmagnetic region to the relative orientation of their magnetization vectors is a fundammental physical phenomenon with a huge impact in the magnetoelectronics industry. ${ }^{1}$ The figure of merit is the ratio between the conductance for antiparallel (AP) and parallel (P) relative magnetic orientations of the FM's, $x$ $\equiv G_{\mathrm{AP}} / G_{\mathrm{P}}$, which can be selected with an external magnetic field. Two different conventions are used to characterize the so-called magnetoresistance $(\mathrm{MR}), \mathrm{MR}_{1}=100 \times(1-x) \%$ and $\mathrm{MR}_{2}=100 \times(1 / x-1) \%$. Metallic multilayers with alternated ferromagnetic and nonmagnetic metallic layers display a large MR known as giant MR (GMR) (Ref. 2). MR in FM/ insulator/FM systems is known as tunnel MR (TMR) (Ref. 3) and values of $\mathrm{MR}_{1} \simeq 30 \%$ have been reported. ${ }^{4}$

More recently, a number of groups have studied MR in $\mathrm{Ni}$ nanocontacts (produced by break junctions or electrodeposition), where two sections of a Ni wire are connected through single ${ }^{5-10}$ or multiple ${ }^{11,12}$ nanometer-size contacts. In this arrangement the intermediate region connecting the two bulk FM's has a different geometry but the same chemical composition, in contrast to GMR and TMR systems. Some groups have obtained values of MR going from $10^{2}$ to $10^{4}$ $\left(x \simeq 10^{-1}\right.$ to $\left.x \simeq 10^{-3}\right)$ (Refs. 5-7 and 12) while others obtain moderate or even negative values. ${ }^{9,10}$ In these systems the resistance predominantly comes from the region with the smallest section, where electron transport is coherent and conductance is dominated by the quantum-mechanical transmission of electrons at the Fermi level. ${ }^{13}$ Mainly, two different mechanisms have been proposed so far to account for the large values of MR when observed: Domain-wall (DW) scattering ${ }^{14}$ and magnetostriction. ${ }^{8,11}$ For the former it has been argued that in the AP arrangement a DW is pinned at the nanocontact ${ }^{15}$ and is responsible for strong spin scattering which gives an extra contribution to the resistance as compared to the $\mathrm{P}$ configuration, resulting in a large "ballistic" MR (Ref. 14).

The fundamental question of whether MR is dramatically enhanced in atomic-sized ferromagnetic contacts due to the presence of a DW remains open and is the subject of this paper. Three ingredients are essential to answer this question: First, as in the case of nonmagnetic nanocontacts, is the electronic structure of the last atom(s) which determines the number of available transport channels. ${ }^{16}$ Second, is the presence of inhomogeneous magnetization profiles, e.g., a DW, which can induce spin scattering and affect current flow. ${ }^{14,17-19}$ Third, is the atomic structure (geometry) which affects both the electronic and magnetic structures, and thus, the transmission of these channels. Previous theoretical works present mutually conflicting results with methodologies that either used an oversimplified description of $\mathrm{Ni}$ electronic structure ${ }^{18,20,21}$ or idealized geometries. ${ }^{19,22}$ Here we present transport calculations across $\mathrm{Ni}$ nanocontacts describing the electronic, magnetic, and atomic structure with $a b$ initio calculations. ${ }^{23-25}$ Our results lead us to conclude that intrinsic ballistic MR is certainly not large in atomicsize Ni nanocontacts.

Spin-dependent transport formalism. Transport through atomic-size metallic contacts is currently understood in terms of elastic transport of noninteracting quasiparticles through a one-body potential that describes their interaction with the constriction. In this approach, the conductance $G$ is proportional to the quantum-mechanical transmission $\mathcal{T}$ associated with the potential. On the other hand, the spontaneous breaking of the spin degeneracy in transition-metal ferromagnets, which is due to electron-electron interactions, can also be properly understood in terms of a mean-field description, where quasiparticles interact with a spin-dependent selfconsistent potential. Once the self-consistent field is determined for a given geometry, the quantum-mechanical spindependent and energy-dependent transmission probability $\mathcal{T}_{\sigma \sigma^{\prime}}(E)$ can be obtained, and thereby the zero-bias conductance, using Landauer's formula, ${ }^{13}$ is

$$
G=\frac{e^{2}}{h}\left[\mathcal{T}_{\uparrow \uparrow}\left(E_{\mathrm{F}}\right)+\mathcal{T}_{\downarrow \downarrow}\left(E_{\mathrm{F}}\right)+\mathcal{T}_{\uparrow \downarrow}\left(E_{\mathrm{F}}\right)+\mathcal{T}_{\downarrow \uparrow}\left(E_{\mathrm{F}}\right)\right] .
$$

In the above expression we only make explicit the dependence of $\mathcal{T}_{\sigma \sigma^{\prime}}$ on the spin channels, which we assume is well-defined in the leads.

Ab initio cluster embedded calculations. It is an experimental fact that the chemical nature of the contact determines the conductance. ${ }^{16}$ As a rule of thumb, ${ }^{26}$ the conductance of single-atom metallic nanocontacts can be as large as the number of valence orbitals, but, in practice, is never larger than the number of valence electrons. A natural de- 
scription of the problem is in terms of a localized atomic orbital basis, preferebly starting from first principles. ${ }^{27}$ In previous publications $\mathrm{s}^{23-25}$ we have presented a method to perfom $a b$ initio calculations of quantum transport through atomic constrictions and molecules which is based on the code GAUSSIAN (Ref. 27). Our approach has been successful in explaining experimental results in paramagnetic nanocontacts. ${ }^{24,28}$ Here we take it a step further to study systems without spin degeneracy, like ferromagnetic nanocontacts. We solve the problem dividing the system in three different parts: left $(L)$ and right $(R)$ electrodes on one side and the contact region on the other. The spin-dependent onebody Hamiltonian is assumed fixed and homogeneous in the bulk electrodes, but it is determined self-consistently in the contact region subject to the appropriate magnetic boundary conditions.

The density-functional theory (DFT) calculations for the contact region are done with both local spin density approximation (LSDA) and the hybrid functional B3LYP (Ref. 27). The LSDA results are robust against different basis sets and so we rely here on a minimal basis set with a core pseudopotential as described in previous works. ${ }^{23,24}$ On the other hand, the B3LYP functional is more sensitive to the basis set due to its nonlocal exchange contribution. Therefore we employ here an all-electron basis set. ${ }^{29}$ The electrodes are described by means of a semiempirical tight-binding Bethe lattice model. With the appropriate parameters, the Bethe lattice can provide a geometry-independent description of the contacts with a bulk density of states (DOS) which is smoother than the real DOS and mimics an average over both disorder realizations and the actual electrode crystal orientations. Spin-mixing solutions are not considered, i.e., $S_{z}$ is a good quantum number. Thus, the last two terms in Eq. (1) do not give any contribution to the conductance. DW-like configurations are obtained for the adequate magnetic boundary conditions and the constraint $S_{z}=0$.

Results. We restrict ourselves to the study of the last (first) plateau of conductance upon stretching (electrodeposition) as, e.g., in the experiment of Viret et al. ${ }^{9}$ (Sullivan et al. ${ }^{6}$ ). A reference atomic structure of the contact region has been initially taken like that shown in the inset of Fig. 1. Following Viret et al., ${ }^{9}$ we consider the narrowest (and most important) region to consist of two pyramids facing each other, formed along the (001) direction, and with the two tip $\mathrm{Ni}$ atoms 2.6- $\AA$ apart forming a dimer. Bulk atomic distances and perfect crystalline order are assumed otherwise. A $b$ initio simulations of the breaking process as the one shown in Fig. 2 support this choice. We stress that the section of the nanocontacts varies in the direction of the current flow. This is the situation in real nanocontacts and differs from perfect onedimensional systems, studied in Refs. 22, and from bulk systems studied by van Hoof et al. ${ }^{19}$ In this regard, the geometries proposed by Bagrets et al..$^{30}$ are closer to real nanocontacts, but are not backed up by experiments or simulations.

From the LSDA DOS projected on the tip atoms (not shown) we see that the $s p$ orbitals are spin split by less than $1 \mathrm{eV}$ and that the minority $(m)$ electrons are hybridized with the $d$ levels, which are present at the Fermi energy. As a consequence, the LSDA DOS for the majority $(M)$ electrons

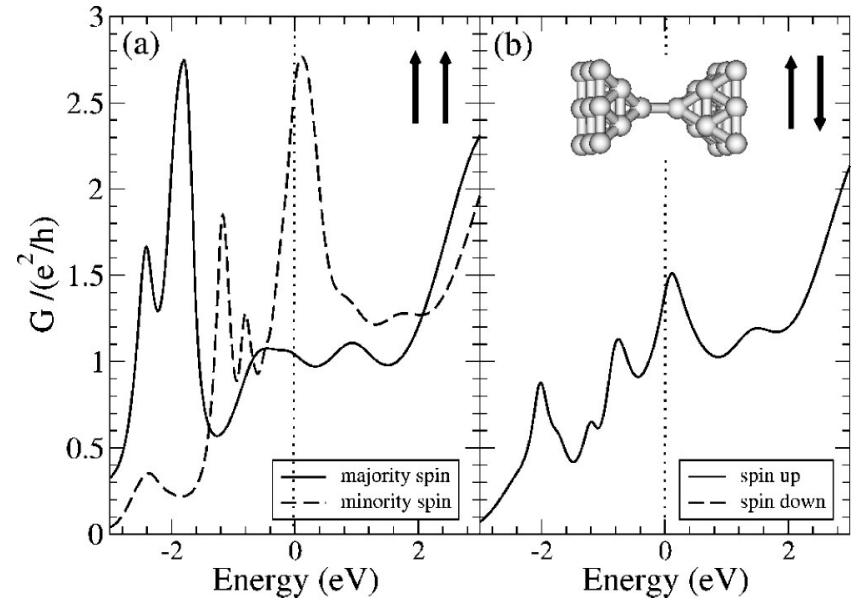

FIG. 1. (a) LSDA conductance per spin channel in the P configuration for the model nanocontact shown in the inset. (b) Same as in (a), but for the AP configuration.

at the Fermi energy is significantly smaller than that for the $m$ ones. These results are compatible with LSDA firstprinciples calculations for systems with translational invariance, ${ }^{22}$ have been properly accounted for in Andersonlike model Hamiltonians, ${ }^{20}$ but are in marked contrast to the $J_{s d}$ model, usually invoked to understand large values of the MR in nanocontacts. ${ }^{14}$ In these models the transmission of itinerant $s$ electrons is perfect in the ferromagnetic case while the $d$ electrons do not contribute to the current since they are localized. The MR depends thus dramatically on the ratio between the spin splitting of the conducting $s$ electrons and the Fermi energy when a DW is present. A large spin splitting needed to give large MR is, however, at odds with the actual Ni band structure and this model must be ruled out from the outset to account for large MR in Ni nanocontacts.

Figure 1 shows the LSDA conductance as a function of energy for both up and down spin channels in two situations: (a) Parallel (P) and (b) antiparallel (AP) bulk magnetic ar-

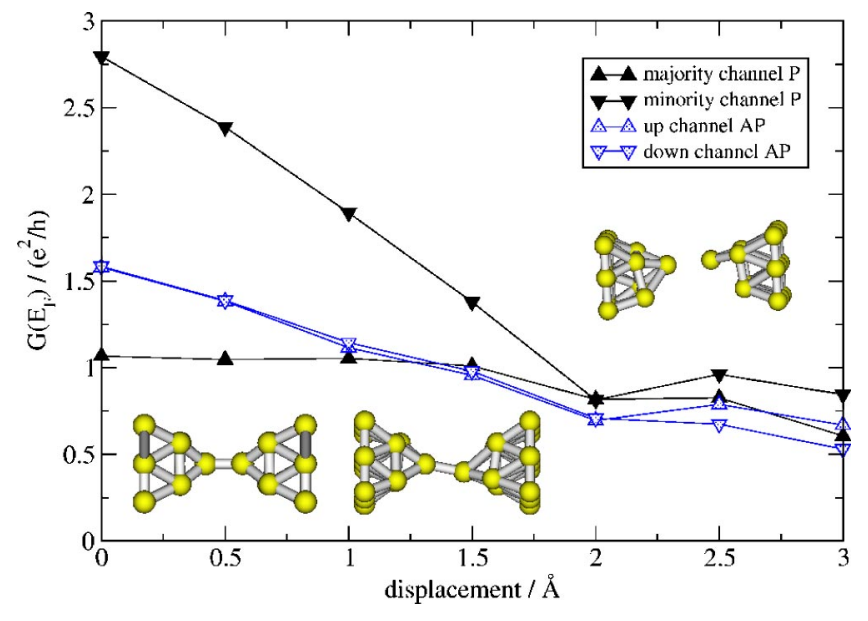

FIG. 2. (Color online) Conductance of both spin channels for the $\mathrm{P}$ and AP configuration as a function of the stretching. The lines connecting the points are just to guide the eye. The insets show the relaxed contact geometry at different values of the displacement $(0$, 1.5 , and $3 \AA$ from left to right). 
rangements. In both cases the self-consistent solution has been forced to respect the high symmetry of the nanocontact. In the AP case the self-consistent magnetization reverses abruptly between tip atoms. The resulting magnetic moment for the contact atoms is $\approx 1.0 \mu_{\mathrm{B}}$ in both situations. This value is significantly larger than that obtained for bulk or surface atoms $\left(\approx 0.6 \mu_{\mathrm{B}}\right)$ and reflects the low coordination of the tip atoms forming the contact. In the $\mathrm{P}$ case the $M$ channel is, for the most part, composed of a single $s p$ orbital channel and conducts perfectly around the Fermi energy (set to zero) while the $m$ channel is composed of three orbital channels (one $s p$ like and two $d$ like, which conduct roughly the same), and exhibits a transmission strongly dependent on the scattering energy. In the AP case the system is invariant under the combined transformations that exchange $L$ with $R$ and $\uparrow$ with $\downarrow$, resulting in identical values for the conductance of the two spin channels, which now are composed of a dominant $s p$ channel and a strongly diminished contribution of the $d$ channels. The conductance ratio for this particular case is $x=2.8 / 3.65=0.77$. This yields $\mathrm{MR}_{1}=23 \%$ and $\mathrm{MR}_{2}=30 \%$, which is clearly below large MR claims. ${ }^{5,6}$

LSDA provides a commonly accepted description of the electronic structure of bulk and surface ferromagnetism in transition metals. ${ }^{29}$ However, the low coordination of atoms in nanocontacts might give rise to a further localization of the $d$ electrons (compared with bulk) and an increase of the magnetic moment. Since LSDA fails to describe properly localized electrons due to the self-interaction problem, various alternatives have been proposed to overcome this problem, ${ }^{31}$ the most popular being the local-density approximation (LDA) plus Hubbard $U$ method (LDA+U) (Ref. 32) and the self-interaction corrected (SIC) LDA scheme (Ref. 33). We should point out that, while the results for the conductance reflect the DOS and look plausible, the fact that reported conductance histograms never show the lowest peak around $4 e^{2} / h$ at high magnetic fields ${ }^{34}$ makes us suspect that either the chosen model for the atomic structure is not realistic or that the electronic structure given by LSDA, due to the problems mentioned above, does not provide the best approximation for nanocontacts. We explore below both possibilities separately.

An alternative approach to the electronic structure comes from the use of a hybrid functional like B3LYP which is a combination of Hartree-Fock and LSDA. The former is free from the self-interaction problem, but fails to include correlation, which is provided by the latter. B3LYP happens to give a very good description of the electronic structure and local magnetic moments in $\mathrm{NiO}$ (Ref. 35) and $\mathrm{La}_{2} \mathrm{CuO}_{4}($ Ref. 36). With B3LYP the results for the conductance (see Fig. 3) are remarkably different in regard to the $m$ channel. Now the $d$ channels give a much smaller contribution to the transmission at the Fermi energy. In this case the MR is negative and its absolute value is even smaller $\left(\mathrm{MR}_{1}=-11 \%\right)$ than the one obtained with LSDA. With B3LYP the bulk and surface magnetic moments are slightly higher than the LSDA ones while the magnetic moment for the tip atoms is roughly the same $\left(\approx 1 \mu_{\mathrm{B}}\right)$.

Since the $m$ conductance evaluated at the LSDA level exhibits a strong dependence on the scattering energy, we study now whether or not different geometries can change

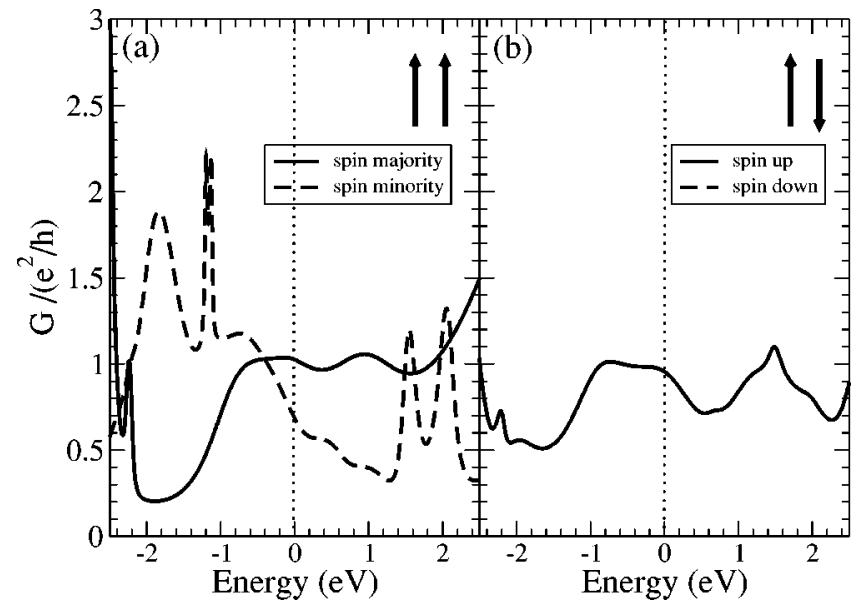

FIG. 3. (a) B3LYP conductance per spin channel in the P configuration for the model nanocontact shown in the inset of Fig. 1. (b) Same as in (a), but for the AP configuration.

the above results qualitatively. In an attempt to explore other realizations of the self-consistent potential compatible with the magnetic boundary conditions and the experimental information, we perform $a b$ initio structural relaxations as a function of the displacement between outer planes in the core cluster. To do so, we consider a cluster like that shown in Fig. 1. The inner atoms in the cluster (10 in total) are allowed to relax to local minimum energy configurations as we stretch. This results, logically, in lower energy solutions and in the loss of symmetry, so that the transmission in the AP case now becomes slightly spin dependent. In Fig. 2 the conductance at the Fermi energy per spin channel for the $\mathrm{P}$ and the AP configurations are shown as a function of the stretching up to the breakup point, starting from a slightly compressed nanocontact. From this figure we see that the conductance of the $m$ channel for the $\mathrm{P}$ configuration changes significantly upon small changes. The MR, on the contrary, barely changes as the nanocontact is stretched and is small, reaching vanishing values for the last points in Fig. 2 . The conductance approaches a stable value around $2 e^{2} / h$ for both $\mathrm{P}$ and AP configurations.

Discussion and conclusions. As mentioned earlier, the maximum number of conducting channels in atomic-size contacts is roughly determined by the number of valence electrons of the contact atom(s). However, as shown above, this hypothetical upper limit is never reached, particularly for the $m$ electrons, remaining essentially only one $M$ channel and one $m$ channel transmitting in the $\mathrm{P}$ case for stretched contacts. This result is impossible to predict without a full atomistic self-consistent calculation. The $M$ channel is $s p$ type. Thus, this channel transmits almost perfectly and evolves smoothly with the stretching of the contact giving a stable contribution $\mathcal{T}_{\uparrow \uparrow} \approx 1$ (see Fig. 2). The $s p$ orbitals in the $m$ channel are strongly hybridized with $d$ orbitals and, therefore, are more sensitive to the contact geometry. The contribution to the conductance of the latter, which form narrower bands, disappears with the stretching and disorder, as expected (see Fig. 2). On the other hand, in the AP configuration mostly one $s p$ orbital channel per spin contributes. The conductance per spin channel lies thus in the vicinity of 
$e^{2} / h$, giving $\approx 2 e^{2} / h$ in total and is fairly stable during the last stage of the breaking of the nanocontact.

To conclude, the reason behind the very small MR values is the orbital (or geometric) blocking of most of the a priori available $m$ channels in the $\mathrm{P}$ configuration due to the nonideal geometry of the nanocontacts. The number of bands at the Fermi energy in the case of a perfect monostrand infinite ferromagnetic chain ${ }^{22}$ is much larger than the number of nonzero eigenvalues of the transmission matrix in a nanocontact (for a given basis and functional). This phenomenon affects mainly the $d$ bands (no DW involved) and, therefore, we call it orbital blocking. On the other hand, $s p$ bands are less sensitive to geometry. In fact, there can only be a significant and positive MR in the cases where the $m$ channel in the $\mathrm{P}$ configuration conducts appreciably. This is the case for the highly symmetric nanocontact presented in Fig. 1 within LSDA and, e.g., for the Ni chains studied in Refs. 22. In the $\mathrm{P}$ case, the number of $M$ and $m$ bands at the Fermi energy is 1 and 6 , respectively. When a DW is formed in the chain, 5 out of $6 \mathrm{~m}$ channels are blocked, remaining 1 per spin, almost fully transmitting. As a result, one expects a ratio $x$ $\simeq 2 / 7$ resulting in $\mathrm{MR}_{2} \simeq 250 \%$ for the ideal chain. ${ }^{22}$ The blocking of a number of channels is due to the fact that the $d$ electrons, and not the $s p$ electrons, are spin split. This could explain some early results, ${ }^{5}$ but not recent ones. ${ }^{6}$ Furthermore, to date, no evidence of chain formation in $\mathrm{Ni}$ has been reported. Even so, scattering at the electrode-chain contact will always be present. For completeness, we have also per- formed calculations for $\mathrm{Ni}$ chains using the B3LYP functional. The number of bands crossing the Fermi energy is now reduced to $1+4$ compared to the LSDA results. This agrees with recent $\mathrm{LSDA}+\mathrm{U}$ calculations reported by Wierzbowska et al. ${ }^{31}$ where the two degenerate flat minority bands $d_{x y}, d_{x^{2}-y^{2}}$ are shifted downwards in energy because of the exchange interaction canceling part of the self-interaction of the strongly localized electrons in these flat bands. However, this is not the reason for the drop in the $m$ conductance seen in Fig. 3 since the corresponding channel does not contribute to the conductance in the LSDA case either. In addition, noncollinear DW's, not considered here, also reduce the MR (Ref. 37). It is our belief that when observed, large values of the MR in Ni nanocontact might be due to magnetostriction effects and the corresponding formation of wider section contacts or to the presence of adsorbates, which modify the local electronic structure. ${ }^{34}$

We acknowledge E. Louis, C. Untiedt, J. A. Vergés, and G. Chiappe for fruitful discussions. J.J.P. acknowledges financial support from Grants No. 1FD97-1358 (FEDER funds) and MAT2002-04429-C03 (MCyT). D.J. acknowledges financial support from MECD under Grant No. UAC2004-0052. J.F.R. acknowledges financial support from Grants No. MAT2003-08109-C02-01 (MCyT), UA/ GRE03-15 (Universidad de Alicante), and Ramon y Cajal Program (MCyT).
${ }^{1}$ S. A. Wolf et al., Science 294, 1488 (2001).

${ }^{2}$ M. N. Baibich et al., Phys. Rev. Lett. 61, 2472 (1988).

${ }^{3}$ M. Julliere, Phys. Lett. 54A, 225 (1975).

${ }^{4}$ J. S. Moodera et al., Phys. Rev. Lett. 74, 3273 (1995).

${ }^{5}$ N. García et al., Phys. Rev. Lett. 82, 2923 (1999).

${ }^{6}$ M. R. Sullivan et al., Phys. Rev. B 71, 024412 (2005).

${ }^{7}$ S. H. Chung et al., Phys. Rev. Lett. 89, 287203 (2002).

${ }^{8}$ M. Gabureac et al., Phys. Rev. B 69, 100401(R) (2004).

${ }^{9}$ M. Viret et al., Phys. Rev. B 66, 220401(R) (2002).

${ }^{10}$ H. Oshima and K. Miyano, Appl. Phys. Lett. 73, 2203 (1998); T. Ono et al., ibid. 75, 1622 (1999).

${ }^{11}$ W. F. Egelhoff, Jr. et al., J. Appl. Phys. 95, 7554 (2004).

${ }^{12}$ H. D. Chopra and S. Z. Hua, Phys. Rev. B 66, 020403(R) (2002); S. Z. Hua and H. D. Chopra, ibid. 67, 060401(R) (2003).

${ }^{13}$ S. Datta, Electronic Transport in Mesoscopic Systems (Cambridge University Press, Cambridge, UK, 1995).

${ }^{14}$ G. Tatara et al., Phys. Rev. Lett. 83, 2030 (1999); H. Imamura et al., ibid. 84, 1003 (2000).

${ }^{15}$ P. Bruno, Phys. Rev. Lett. 83, 2425 (1999).

${ }^{16}$ N. Agraït et al., Phys. Rep. 377, 81 (2003), and references therein.

${ }^{17}$ P. M. Levy and S. Zhang, Phys. Rev. Lett. 79, 5110 (1997).
${ }^{18}$ G. Tatara and H. Fukuyama, Phys. Rev. Lett. 78, 3773 (1997).

${ }^{19}$ J. B. A. N. van Hoof et al., Phys. Rev. B 59, 138 (1999).

${ }^{20}$ A. R. Rocha and S. Sanvito, Phys. Rev. B 70, 094406 (2004).

${ }^{21}$ J. Velev and W. H. Butler, Phys. Rev. B 69, 094425 (2004).

${ }^{22}$ A. Smogunov et al., Surf. Sci. 507, 609 (2002); 532, 549 (2003).

${ }^{23}$ J. J. Palacios et al., Phys. Rev. B 64, 115411 (2001).

${ }^{24}$ J. J. Palacios et al., Phys. Rev. B 66, 035322 (2002).

${ }^{25}$ E. Louis et al., Phys. Rev. B 67, 155321 (2003).

${ }^{26}$ E. Scheer et al., Nature (London) 394, 154 (1998).

${ }^{27}$ M. J. Frisch et al., GAUSSIAN 03, Revision B.01 (Gaussian, Inc., Pittsburgh, PA, 2003).

${ }^{28}$ Y. García et al., Phys. Rev. B 69, 041402(R) (2004).

${ }^{29}$ K. Doll, Surf. Sci. 544, 103 (2003).

${ }^{30}$ A. Bagrets et al., Phys. Rev. B 70, 064410 (2004).

${ }^{31}$ M. Wierzbowska et al., Phys. Rev. B (to be published).

${ }^{32}$ V. I. Anisimov et al., Phys. Rev. B 44, 943 (1991).

${ }^{33}$ J. P. Perdew and A. Zunger, Phys. Rev. B 23, 5048 (1981).

${ }^{34}$ C. Untiedt et al., Phys. Rev. B 69, 081401(R) (2004).

${ }^{35}$ I. P. R. Moreira et al., Phys. Rev. B 65, 155102 (2002).

${ }^{36}$ J. K. Perry et al., Phys. Rev. B 63, 144510 (2001).

${ }^{37} \mathrm{D}$. Jacob et al. (unpublished). 